\title{
Histochemical Patterns of Collagenic Fibers in the Benign and Malignant Breast Lesions
}

\author{
Abdulbaset Mohamed Elasbali \\ Department of Clinical Laboratory Science Department, College of Applied Medical Science, Jouf University, Qurayyat, \\ Saudi Arabia \\ Email: elasbaliuoh@gmail.com
}

How to cite this paper: Elasbali, A.M. (2019) Histochemical Patterns of Collagenic Fibers in the Benign and Malignant Breast Lesions. Advances in Breast Cancer Research, 8, 1-10.

https://doi.org/10.4236/abcr.2019.81001

Received: November 1, 2018

Accepted: December 11, 2018

Published: December 14, 2018

Copyright (C) 2019 by author and Scientific Research Publishing Inc. This work is licensed under the Creative Commons Attribution International License (CC BY 4.0).

http://creativecommons.org/licenses/by/4.0/

\section{(c) (i) Open Access}

\begin{abstract}
Cancer is one of the leading causes of death worldwide and breast cancer is the most commonly diagnosed cancer among women. An increased incidence of different types of breast cancer has been reported. This study was designed to evaluate the different types of breast cancer and its possible risk of neoplasmic transformation to an advanced malignant stage from a benign tumor. The histochemical patterns of collagen fibers in the benign and malignant breast lesions were evaluated. From the 50 tissue samples, 25 were malignant breast lesions and 25 were benign breast tumor. Hematoxylin and Eosin (HE), Van Gieson staining were performed to detect a benign and malignant tumor as well as collagen fibers. We found that significant cases after age of 35 were associated with ductal carcinoma while most of the cases within the age of 25 years were associated with fibrocystic changes. The intensity of collagen fiber was higher to Ductal Carcinoma while negative and less intense for Fibroblastic changes. Furthermore, a consistent association of other lesions, such as Lobular Carcinoma, Fibroadenoma, Papilloma and Fat necrosis and noticeable staining for collagen was observed for the different lesion. Our study suggested that women with age of 25 with benign lesion of fibrocystic change and ductal carcinoma are highly susceptible to develop advanced malignant tumor with age. Therefore, quantitative measurement of collagen fiber and regular follow-up are recommended to avoid the possible risk of developing advanced malignant lesions.
\end{abstract}

\section{Keywords}

Cancer, Collagen, Malignant Lesions, Benign Tumor

\section{Introduction}

Breast cancer is the first tumor disease among women, causing more than 
600,000 new cases per year [1]. Furthermore, it is also the most common cancer and the principal cause of cancer death among females worldwide [2]. In addition, breast cancer is the second leading cause of cancer death among women, causing more than 39,000 deaths every year in the United States alone [3]. In 2015, cancer was accounting for 429.16 million new cases and 281.42 million deaths in China [4] [5]. Although in the last years, the early diagnosis of this disease has improved overall survival [6].

According to Cancer Research UK, more than $90 \%$ of women diagnosed with breast carcinoma at the earliest stage survive for at least 5 years compared to about $15 \%$ of women diagnosed with the most advanced stage of cancer [7] [8]. Breast cancer remains a very serious problem for public health and there are still many research areas to open.

The extracellular matrix (ECM) of the tumor plays an important role in the development of cancer through its ability to modify some of the vital signals perceived by cancer cells associated with cancer [9]. Experimental evidence has demonstrated that the structure and composition of the extracellular matrix (ECM) of tumor-affected breast tissue undergoes alteration [10], especially its collagenous component [11]. Collagen is a major component of the ECM and is increasingly recognized to play a critical role in regulating breast cancer progression. Although most research on collagen in breast cancer has focused on type I collagen (Col1), and many reports have documented a negative correlation between Coll expression and prognosis in breast cancer patients, collagen types IV, V, VI, and XVIII have also been implicated in modulation of breast cancer cell activities and fate [12] [13]. There are many types of breast cancer; most common types are ductal carcinoma in situ, invasive ductal carcinoma and invasive lobular carcinoma. Most breast cancers are carcinomas. There are other less common types of breast cancers, such as sarcomas, phyllodes, Paget disease, and angiosarcomas which start in the cells of the muscle, fat, or connective tissue [14].

However, there is a frequent intervening between these different breast cancer entities. Also, the components of some of these malignant lesions mimic the components of some benign breast lesions. Consequently, sometimes, the precise diagnosis requires the application of extra more sophisticated techniques, which might not be available in some settings, particular for inclusion and exclusion criteria for some researches. Thus, there is a need for an easy and cost-effective method to fulfill this. Therefore, the present study aimed to assess the histochemical pattern of collagen fibers in the benign and malignant breast lesions, to use it to classify breast lesions, as well as, to stimulate the future concept of changing the qualitative consideration of collagen fibers to quantitative measurement.

\section{Materials and Methods}

This study involved 50 samples collected from women with breast lesions. All 
samples were in the form of formalin fixed paraffin tissue blocks. Out of 50 samples, 25 were malignant breast lesions, and 25 were benign breast tumor. The tissue samples were collected from histopathology laboratory in Ribat University Hospital, Khartoum state-Sudanin December 2017. The sample included a whole coverage of malignant samples within one year in one diagnostic laboratory and random selection of 25 benign samples regardless of diagnosis. Master sheets were used to retrieve all patients records and sample data; age and type of breast cancer. Two sections of $4 \mu$ in thickness were cut from each block using Leica microtome (Leica Microsystems, NussiochGmbll, model: RM 2125RT, ser NO. 8843/04-2005-China). Then each section was floated in 70\% ethanol and water bath (Electrothermalser NO. 18861434-China) at 40c0, consecutively. Each floated section was mounted on frosted end slide. All slides contained sections were dried in a dry oven (WTC binder 7200 TUTTLINGEN, B28, NO. 88485 -USA) at $60 \mathrm{c0}$ for 30 minutes. Subsequently, one section was stained applying Hematoxylin and Eosin ( $\mathrm{H} \& \mathrm{E})$ staining to confirm the diagnosis and the other one was stained by Van-gieson staining to detect collagen fiber. H\&E (Mayer's) and Van-gieson staining methods were achieved according to standard procedure technique, described in [15].

\subsection{Ethical Consent}

The study was approved by Ethical Review Board, College of Applied Medical Science, University of Hail, Saudi Arabia. In addition to the fact, the authors followed the tenants of the Declaration of Helsinki.

\subsection{Statistical Analysis}

For all statistical analyses including frequencies and cross-tabulations, the SPSS statistical software version 16 was used. Pearson chi-square test was used and P. values of 0.05 or less were regarded as statistically significant.

\section{Results}

This study investigated 50 patients with different breast lesions, their ages ranging from 17 to 85 years with a mean age of 39 years. All study subjects were females. With regard to the age of the patients, the majority of patients were at age group $\leq 25$ years constituting 13/50 (26\%) followed by age range $36-45$ years representing 12/50 (24\%). The majority of patients with benign lesions were at age group $\leq 25$ years followed by $26-35,46-55$ and $36-45$ constituting 12/25 (48\%), 5/25 (20\%), 4/25 (16\%) and 3/25 (12\%), respectively. For the patients with malignant lesions the majority were found at age group 36 - 45 years followed by 56+, 26 - 36 and 36 - 45 years representing 9/25 (36\%), 8/25 (32\%), $4 / 25$ (16\%), and 3/25 (12\%), respectively, as indicated in Table 1, Figure 1.

With regard to the breast lesion type, about 21/50 (84\%) the malignant lesions were ductal carcinoma and the remaining 4/25 (16\%) were lobular carcinoma. Moreover, the majority of benign lesions were fibrocystic changes constituting 
$19 / 25$ (76\%) followed by fibroadenoma representing 4/25 (16\%), as indicated in Table 2, Figure 2. On the other hand, most of ductal carcinoma cases were found at age group 36 - 45 years and equally in 56+ years representing 7/21 $(33.3 \%)$ for each. Half (50\%) cases of lobular carcinoma were found at age range 36 - 45 years. The great majority of fibrocystic changes were found at age group $\leq 25$ years representing $9 / 19(47.4 \%)$. About $2 / 4$ (\%) and 2/4 (\%) of the cases of fibroadenoma were found in age ranges, $\leq 25$ years and 26 - 35 years, correspondingly, as shown in Table 2, Figure 2.

Table 3 summarized the distribution of benign and malignant lesions by the intensity of collagen fibers. In general, the presence of collagen fibers in breasts carcinomas compared to other benign breast lesions was statistically significant, the $95 \%$ confidence intervals $(95 \% \mathrm{CI})$ and the Odd Ratio (OR): OR (95\% CI) = 9.3333 ( 1.0523 to 82.7832 ), $\mathrm{P}=0.0449$. The majority of ductal carcinoma tissues were found with ++ collagen intensity. Hence, the majority of lobular CA was found with + intensity, as shown in Figure 3, Photomicrographs 1-3.

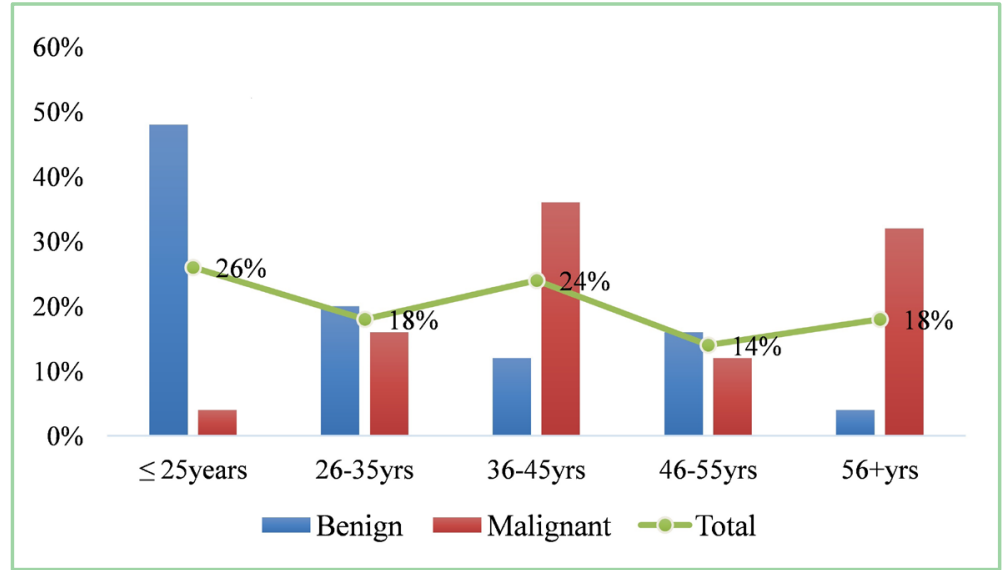

Figure 1. Description of the study subjects by age and lesions type.

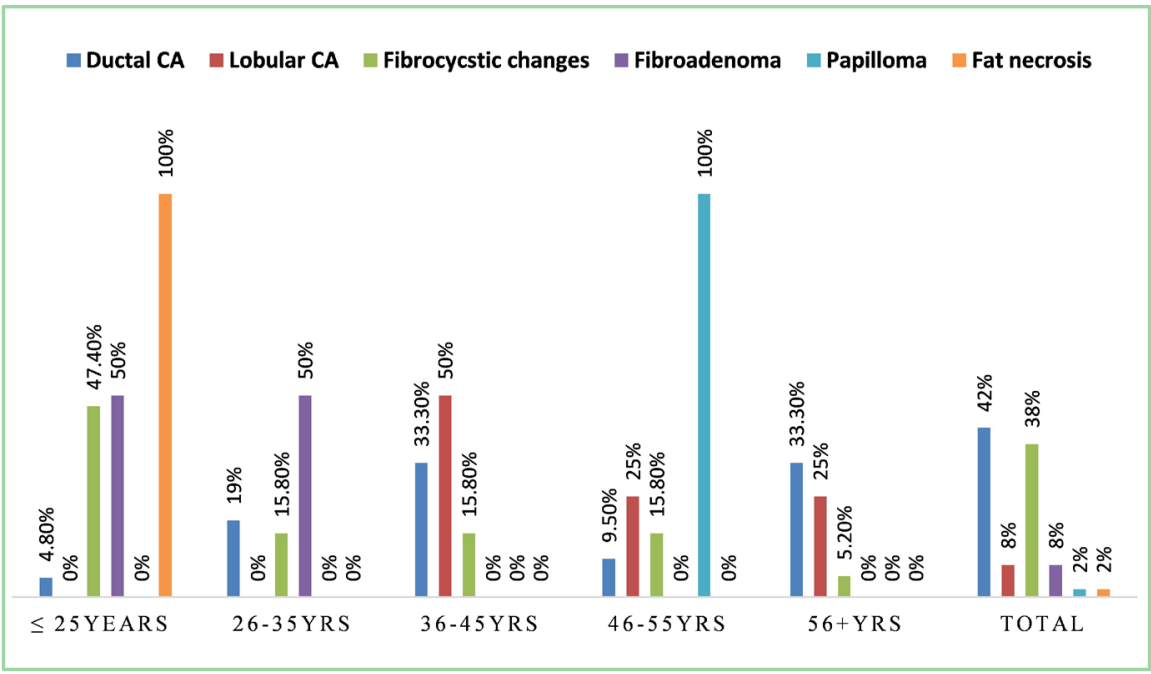

Figure 2. Description of the study subjects by age and benign and malignant lesions type. 


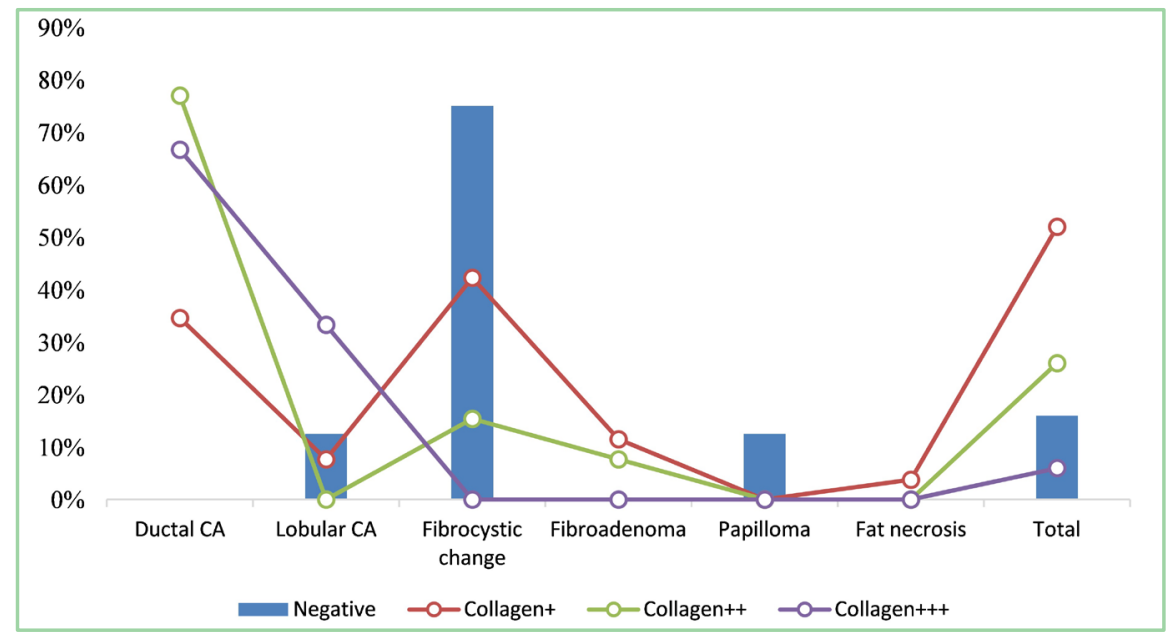

Figure 3. Description of benign and malignant lesions by the intensity of collagen fibers.

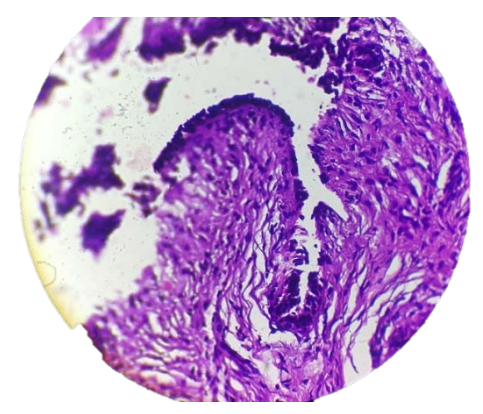

Photomicrograph 1. Section obtained from patient with breast cancer, $\mathrm{H}$ \& E staining $100 \times$.

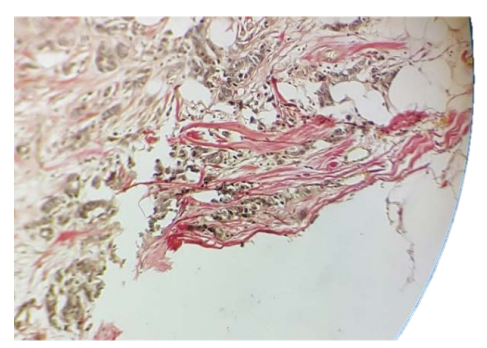

Photomicrograph 2. Section obtained from the patient with breast cancer, showing dese collagen fibers (+) Van-gieson staining $100 \times$.

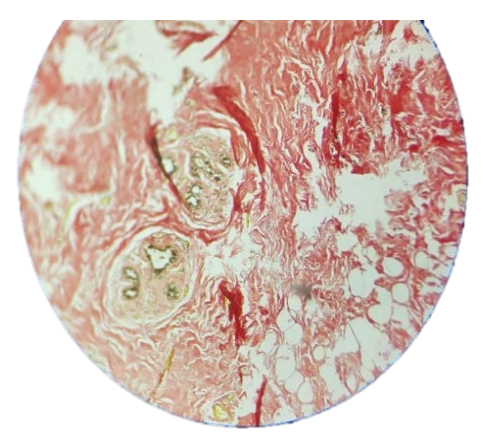

Photomicrograph 3. Section obtained from patient with breast cancer, showing dese collagen fibers $(+++)$ Van-gieson staining 100×. 
Table 1. Distribution of the study subjects by age and lesion type.

\begin{tabular}{cccc}
\hline Age group & Benign lesions & Malignant lesions & Total \\
\hline$\leq 25$ years & 12 & 1 & 13 \\
$26-35$ & 5 & 4 & 9 \\
$36-45$ & 3 & 9 & 12 \\
$46-55$ & 4 & 3 & 7 \\
$56+$ & 1 & 8 & 9 \\
Total & 25 & 25 & 50 \\
\hline
\end{tabular}

Table 2. Distribution of the study subjects by age and benign and malignant lesions type.

\begin{tabular}{ccccccc}
\hline Lesion's type & $\leq 25$ years & $\mathbf{2 6 - 3 5}$ & $\mathbf{3 6 - 4 5}$ & $\mathbf{4 6 - 5 5}$ & $\mathbf{5 6 +}$ & Total \\
\hline Ductal carcinoma & 1 & 4 & 7 & 2 & 7 & 21 \\
Lobular carcinoma & 0 & 0 & 2 & 1 & 1 & 4 \\
Fibrocystic changes & 9 & 3 & 3 & 3 & 1 & 19 \\
Fibroadenoma & 2 & 2 & 0 & 0 & 0 & 4 \\
Papilloma & 0 & 0 & 0 & 1 & 0 & 1 \\
Fat necrosis & 1 & 0 & 0 & 0 & 0 & 1 \\
Total & 13 & 9 & 12 & 7 & 9 & 50 \\
\hline
\end{tabular}

Table 3. Distribution of benign and malignant lesions by the intensity of collagen fibers.

\begin{tabular}{cccccc}
\hline Lesion Type & \multicolumn{5}{c}{ Collagen Intensity } \\
\hline Negative & + & ++ & +++ & Total \\
\hline Ductal CA & 0 & 9 & 10 & 2 & 21 \\
Lobular CA & 1 & 2 & 0 & 1 & 4 \\
Fibrocystic changes & 6 & 11 & 2 & 0 & 19 \\
Fibroadenoma & 0 & 3 & 1 & 0 & 4 \\
Papilloma & 1 & 0 & 0 & 0 & 1 \\
Fat necrosis & 0 & 1 & 0 & 0 & 1 \\
Total & 8 & 26 & 13 & 3 & 50 \\
\hline
\end{tabular}

\section{Discussion}

Breast cancer is the most common cancer diagnosed among women worldwide. It is also the second leading cause of death among women after lungs cancer. Scientist, clinician and physician are putting huge efforts to overcome the incidences related to the all types of cancer and breast cancers as well. Breast cancer possesses a huge challenge to find out the effective molecular target because of its diverse types and complicated nature [16]. Fortunately, significant improvements have been made in order to effective diagnostic and treatment of breast cancer, in this report, we aimed to screen the 50 breast cancer patients visited Ribat University Hospital, Khartoum State-Sudan. Various parameters such as 
HE was used to confirm malignant tumor and Van-gieson was to detect collagen fiber. Furthermore, we analyzed on the basis of benign and malignant nature as well as its association with different age groups. We further analyzed the benign and malignant tumor with its intensity of collagen fibers and lesion types. I found that young women age of below 25 are susceptible to develop benign breast tumor and malignant lesions while the women above the age 35 are more are susceptible for malignant lesion more in compare to young women below 25 . Our result also demonstrated that women up to the age of 45 are more risk to fibrocytic changes and fibroadenoma while women over the age of 45 are more susceptible to ductal carcinoma. While looking at the distribution of benign and malignant lesions by the intensity of collagen fibers we found that most of the cases were positive to collagen intensity related to ductal carcinoma. In contrast, approximately similar cases collagen negative was observed which was associated with fibrocystic changes. An increase in the incidence of ductal carcinoma of the breast has been documented, and concerns regarding overly aggressive treatment have been raised. The incidence of ductal carcinoma in situ (DCIS) of the breast has risen dramatically in the United States. Before 1980, DCIS was rarely diagnosed [17] but currently more than $14 \%$ of breast cancers diagnosed in the United States are DCIS. About one in every 1300 screening mammographic examinations will lead to the diagnosis of DCIS [18]. According to our study most of the case after the age of 25 is reported to develop ductal carcinoma and with growing age women displayed significantly high risk to develop this DC's. Similarly, Lobular carcinoma is also displayed a risk factor with growing age, these findings are consistent with the growing incidents of the ductal carcinoma in women after the age of 35. Fibrocystic changes (FCCs) are the most frequent benign conditions of the breast, diagnosed in $50 \%$ of women examined clinically and in $90 \%$ of women in histopathological studies. These benign disorders have two important implications from breast cancer diagnosis and management. First, FCCs can mimic breast cancer on clinical examination, mammography and breast ultrasonography, leading to unnecessary breast biopsies and patient anxiety. Second, some types of FCCs represent a risk factor for the subsequent development of breast cancer [19]. Numerous studies have demonstrated the clinical importance of FCCs, related to the high prevalence of the condition, the considerable impact on quality of life and the increased breast cancer risk for women with proliferative lesions [20]. In our study, we found that most of the case under the age of 25 are reported to develop Fibrocystic changes and out of 50 patients total 19 patients reported fibrocystic changes, among them 9 women were under age of 25 which suggests that young women are a high risk of developing FCs in this population.

Collagen is an important protein within the extracellular matrix (ECM) of normal and malignant tissues. In normal breast tissue, increased collagen is associated with an increased risk of developing breast cancer and increased disease aggressiveness. Recent studies of intra-tumor collagen demonstrate that collagen 
fiber alignment and density associated with aggressive disease and increased invasion [11]. Furthermore, a high density of interconnected collagen fibers within the ECM of tumors can block the diffusion of drugs into the tumor [21]. In our experiment, we observed different cases with collage positive and negative. Most patients with ductal CA were collagen positive however the cases with fibrocystic changes found collagen negative and less intensely positive. Other cases such as Lobular CA and Papilloma were also collagen negative; these results supported the notion ongoing detection of the collagen in the malignant tissue are an indication of malignant breast tumor.

Our study has several limitations. First, it was conducted on population-based data, and the volume of the cases are also limited information of patients, and no information of ongoing treatment therapy was provided, in addition, there was no information of tumor stage and migration status. However, detail information of detail information of age groups, identification of different lesion types (Ductal carcinoma, Lobular carcinoma, fibrocystic changes, Fibroadenoma, Papilloma and Fat necrosis), and distribution of benign and malignant lesions by the intensity of the collagen fibers, and clear staining for HE, collagen provided adequate information about the risk of different types of breast cancers to the women in different age groups. With growing age, a screening test for the breast cancer is also recommended a cases positive with doctoral carcinoma and fibrocystic changes are needed to follow up the treatment as there are high chances of malignant transformation from the benign state with age.

In summary, from our study, we can conclude that young women within the age of 25 are prone to develop benign while with they are higher risk to develop a malignant lesion. Also, young women under the age of 25 found to develop collagen negative Fibrocystic changes compared to the women with age of 35 or more found to be suffered from ductal carcinoma that was collagen positive. Our study provides the insight of the possible risk of different types of breast cancer associated with different types of age groups leading to provide novel parameter for effective treatment, diagnostic and management of women breast cancers. Our study also suggests that the patients who develop Ductal Carcinoma and Fibrocystic changes are at risk to develop, therefore, proper follow up are recommended to avoid further risk.

\section{Conclusion}

Collagen fiber is predominantly present in breast lesions compared to its benign breast lesions and might be helpful in differential diagnosis using cheap conventional methods. The findings of the present may stimulate further research in this context to develop a specific quantitative method for evaluation of collagen fibers in different breast lesions.

\section{Acknowledgements}

The author would like to thank people at Histopathology laboratory in Ribat 
University Hospital, Khartoum State, Sudan for giving the sample. Also, author's deep thanks go to the Department of Pathology, College of Medicine, for providing the facilities for doing the practical work. My endless thank goes to Pro. Hussain Gadelkarim Ahmed, Head of Department of Pathology, College of Medicine, University of Hail, for collaboration and valuable instructions.

\section{Conflicts of Interest}

The author declares no conflicts of interest regarding the publication of this paper.

\section{References}

[1] Siegel, R., Naishadham, D. and Jemal, A. (2012) Cancer Statistics, 2012. CA: Cancer Journal for Clinicians, 62, 10-29. https://doi.org/10.3322/caac.20138

[2] Torre, L.A., Bray, F., Siegel, R.L., Ferlay, J., Lortet-Tieulent, J. and Jemal, A. (2015) Global Cancer Statistics, 2012. CA: Cancer Journal for Clinicians, 65, 87-108. https://doi.org/10.3322/caac.21262

[3] DeSantis, C., Siegel, R., Bandi, P. and Jemal, A. (2011) Breast Cancer Statistics, 2011. CA: Cancer Journal for Clinicians, 61, 409-418. https://doi.org/10.3322/caac.20134

[4] Chen, W., Zheng, R., Baade, P.D., Zhang, S., Zeng, H., Bray, F. and He, J. (2016) Cancer Statistics in China, 2015. CA: Cancer Journal for Clinicians, 66, 115-132. https://doi.org/10.3322/caac. 21338

[5] Ording, A.G., Boffetta, P., Garne, J.P., Nystrom, P.M., Cronin-Fenton, D., Froslev, T. and Lash, T.L. (2015) Relative Mortality Rates from Incident Chronic Diseases among Breast Cancer Survivors-A 14 Year Follow-Up of Five-Year Survivors Diagnosed in Denmark between 1994 and 2007. European Journal of Cancer, 51, 767-775. https://doi.org/10.1016/j.ejca.2015.02.001

[6] Tirona, M.T. (2013) Breast Cancer Screening Update. American Family Physician, 87, 274-278.

[7] Cancer Research UK. (2016) Breast Cancer Survival Statistics 2016. http://www.cancerresearchuk.org/about-cancer/cancer-symptoms/why-is-early-dia gnosis-important

[8] Dutzu, R., Bedwani, R.N., Vana, J., Baker, H.W. and Murphy, G.P. (1980) Noninvasive Breast Carcinoma: Results of a National Survey by the American College of Surgeons. Annals of Surgery, 192, 139-147. https://doi.org/10.1097/00000658-198008000-00001

[9] Lu, P., Weaver, V.M. and Werb, Z. (2012) The Extracellular Matrix: A Dynamic Niche in Cancer. The Journal of Cell Biology, 196, 395-406. https://doi.org/10.1083/jcb.201102147

[10] Burke, K., Tang, P. and Brown, E. (2013) Second Harmonic Generation Reveals Matrix Alterations during Breast Tumor Progression. Journal of Biomedical Optics, 18, Article ID: 031106. https://doi.org/10.1117/1.JBO.18.3.031106

[11] Provenzano, P.P., Inman, D.R., Eliceiri, K.W., Knittel, J.G., Yan, L., Rueden, C.T., White, J.G. and Keely, P.J. (2008) Collagen Density Promotes Mammary Tumor Initiation and Progression. BMC Medicine, 6, 11-16. https://doi.org/10.1186/1741-7015-6-11

[12] Albini, A., Mirisola, V. and Pfeffer, U. (2008) Metastasis Signatures: Genes Regulating Tumor-Microenvironment Interactions Predict Metastatic Behavior. Cancer 
and Metastasis Reviews, 27, 75-83. https://doi.org/10.1007/s10555-007-9111-x

[13] Luparello, C. (2013) Aspects of Collagen Changes in Breast Cancer. Journal of Carcinogenesis and Mutagenesis, S13, 7.

[14] Calhoun, K.E., Allison, K.H., Kim, J.N., et al. (2014) Chapter 62: Phyllodes Tumors. In: Harris, J.R., Lippman, M.E., Morrow, M. and Osborne, C.K., Eds., Diseases of the Breast, 5th Edition, Lippincott-Williams \& Wilkins, Philadelphia, PA.

[15] Bancroft, J.D. and Gamble, M. (2011) Theory and Practice of Histological Techniques. 6th Edition, Churchill Livingstone, Elsevier, Philadelphia, PA, 135.

[16] Pandeya, D.R., Rajbhandari, A., Nepal, M., Abdalhabib, E.K., Bhatta, M., Malla, S.S., Upadhyay, L. and Saiem Al Dahr, M.H. (2018) Comparative Study of Serum Lipid Profiles in Nepalese Cancer Patients Attending a Tertiary Care Hospital. Asian Pacific Journal of Cancer Prevention, 19, 491-495.

[17] Ernster, V.L., Barclay, J., Kerlikowske, K., Wilkie, H. and Ballard-Barbash, R. (2000) Mortality among Women with Ductal Carcinoma in Situ of the Breast in the Population-Based Surveillance, Epidemiology and End Results Program. Archives of Internal Medicine, 160, 953-958. https://doi.org/10.1001/archinte.160.7.953

[18] Kopans, D.B. (2003) Re: Detection of Ductal Carcinoma in Situ in Women Undergoing Screening Mammography. Journal of the National Cancer Institute, 95, 487. https://doi.org/10.1093/jnci/95.6.487

[19] Guray, M. and Sahin, A.A. (2006) Benign Breast Diseases: Classification, Diagnosis, and Management. Oncologist, 11, 435-449.

https://doi.org/10.1634/theoncologist.11-5-435

[20] Dupont, W.D. and Page, D.L. (1985) Risk Factors for Breast Cancer in Women with Proliferative Breast Disease. The New England Journal of Medicine, 312, 146-151. https://doi.org/10.1056/NEJM198501173120303

[21] Jain, R.K. and Stylianopoulos, T. (2010) Delivering Nanomedicine to Solid Tumors. Nature Reviews Clinical Oncology, 7, 653-664. https://doi.org/10.1038/nrclinonc.2010.139 